Transport processes in dense stellar plasmas NAOKI ITOH

Departmant of Physics, Sophia University, 7-1, Kioi-cho, Chiyoda-ku, Tokyo 102, Japan

\begin{abstract}
Transport processes in dense stellar plasmas which are relevant to the interiors of white dwarfs and neutron stars are reviewed. The emphasis is placed on the accuracy of the numerical results. In this review we report on the electrical conductivity and the thermal conductivity of dense matter. The methods of the calculations are different for the liquid metal phase and the crystalline lattice phase. We will broadly review the current status of the calculations of the transport properties of dense matter, and try to give the best instructions available at the present time to the readers.

Nous présentons une revue des propoiétés de transport dans les plasmas denses stellaires caractéristiques des intérieurs de naines blanches et d'étoiles à neutrons. L'accent est mis sur la précision des résultats numériques. Nous présentons la conductivité electrique et la conductivité thermique dans la matière dense. Les méthodes de calcul sont différentes dans la phase liquide et dans la phase cristalline. Nous donnons une revue générale des calculs des propriétés de transport dans la matière dense, et nous essayons de donner les meilleures instructions quant aux données disponibles actuellement.
\end{abstract}

\title{
18.1 Introduction
}

In recent years white dwarf asteroseismology opened up a new fertile land of astrophysics (Bradley \& Winget 1991; Bradley, Winget, \& Wood 1992). Consequently, the basic physics data which go into white dwarf models need 
to be sufficiently accurate that they should live up to the standard required by the asteroseismological data.

In order to match the accuracy required by white dwarf asteroseismology, tremendous effort has been devoted in the recent years to improve the numerical accuracy of the calculations of the transport properties of dense matter. For the liquid metal phase the papers of Itoh et al. (1983) and Mitake, Ichimaru, \& Itoh (1984) have given significantly improved results of the conductivities upon some of the previous results reported in the papers of Flowers \& Itoh (1976), Flowers \& Itoh (1981), and Yakovlev \& Urpin (1981). The paper of Itoh et al. (1984) has significantly improved the results of the conductivities in the cyrstalline lattice phase reported in the papers of Flowers \& Itoh (1976), Flowers \& Itoh (1981), Yakovlev \& Urpin (1981), and Raikh \& Yakovlev (1982). Recently, Itoh, Hayashi, \& Kohyama (1993) have included the lower densities $10^{0}-10^{4} \mathrm{gcm}^{-3}$ for the calculation of the electrical and thermal conductivities of dense matter in the crystalline lattice phase. The impurity scattering contributions to the electrical and thermal conductivities of dense matter in the crystalline lattice phase have been recently recalculated by Itoh \& Kohyama (1993). The viscosity of dense matter in the liquid metal phase has been calculated by Itoh , Kohyama , \& Takeuchi (1987).

\subsection{Electrical and Thermal Conductivities in the Liquid Metal Phase}

In this section we review the calculations of the electrical and thermal conductivities of dense matter in the liquid metal phase following the paper of Itoh et al. (1983). We consider the case that the atoms are completely pressure-ionized. The corresponding condition is expressed by

$$
E_{F}(\rho) \geq Z^{2} \quad \mathrm{Ry},
$$

where $E_{F}(\rho)$ is the electron Fermi level at zero temperature and at a given mass density $\rho$. Numerically, the condition (1) can be rewritten as

$$
\rho \geq 0.378 A Z^{2} \mathrm{gcm}^{-3},
$$

where $Z$ and $A$ are the atomic number and mass number of the atom considered, respectively. Thus we have $\rho \geq 6.05 \mathrm{gcm}^{-3}$ for ${ }^{4} \mathrm{He}, \rho \geq 1.63 \times$ $10^{2} \mathrm{gcm}^{-3}$ for ${ }^{12} \mathrm{C}, \rho \geq 3.87 \times 10^{2} \mathrm{gcm}^{-3}$ for ${ }^{16} \mathrm{O}$, and $\rho \geq 1.43 \times 10^{4} \mathrm{gcm}^{-3}$ for 
${ }^{56} \mathrm{Fe}$. We further restrict ourselves to the density-temperature region in which electrons are strongly degenerate. This condition is expressed as

$$
T \ll T_{F}=5.930 \times 10^{9}\left[\left[1+1.018(Z / A)^{2 / 3} \rho_{6}^{2 / 3}\right]^{1 / 2}-1\right] \quad \mathrm{K},
$$

where $T_{F}$ is the Fermi temperature and $\rho_{6}$ is the mass density in units of $10^{6} \mathrm{gcm}^{-3}$.

For the ionic system, we consider in this section the case that it is in the liquid state. The criterion for that is a subject of the recent debate (see the review paper of H.E. DeWitt in the present Proceedings); but we follow in this review the criterion given by Ogata \& Ichimaru (1987)

$$
\Gamma \equiv \frac{Z^{2} e^{2}}{a k_{B} T}=2.275 \times 10^{-1} \frac{Z^{2}}{T_{8}}\left(\frac{\rho_{6}}{A}\right)^{1 / 3} \leq \Gamma_{m}=180,
$$

where $a=\left[3 /\left(4 \pi n_{i}\right)\right]^{1 / 3}$ is the ion-sphere radius and $T_{8}$ is the temperature in units of $10^{8} \mathrm{~K}$. The analytic fitting formulae presented in the paper of Itoh et al. (1983) are valid for $2 \leq \Gamma \leq \Gamma_{m}$, irrespective of the precise value of $\Gamma_{m}$.

The low-temperature quantum corrections for the ions can be neglected unless the parameter

$$
y \equiv \frac{\hbar^{2} k_{F}^{2}}{2 M k_{B} T}=1.656 \times 10^{-2} \frac{1}{A T_{8}}\left(\frac{Z}{A}\right)^{2 / 3} \rho_{6}^{2 / 3},
$$

where $k_{F}$ is the Fermi wavenumber of the electrons and $M$ is the mass of the ion, becomes appreciable compared with unity. The prescriptions to take into account the low-temperature quantum corrections for the ions have been given in detail by Mitake, Ichimaru, \& Itoh (1984). However, these prescriptions are not complete, because they are powerless for the case $y \geq 1$. This situation is quite ironical, as the correct prescrptions for low-temperature quantum corrections are badly needed for $y \geq 1$. When we can calculate the low-temperature quantum corrections accurately, they are small anyway. Therefore, if one is not worried by the small corrections, it would be probably wise to disregard altogether the low-temperature quantum corrections and use simply the results of Itoh et al. (1983) for the whole range of $2 \leq \Gamma \leq \Gamma_{m}$. Then one would not be too wrong in getting the right behavior of the conductivities. On the other hand, if one uses the results of Mitake, Ichimaru, \& Itoh (1984) for the cases $y \geq 1$, one would be led to unphysical results, because the calculation of Mitake Ichimaru, 


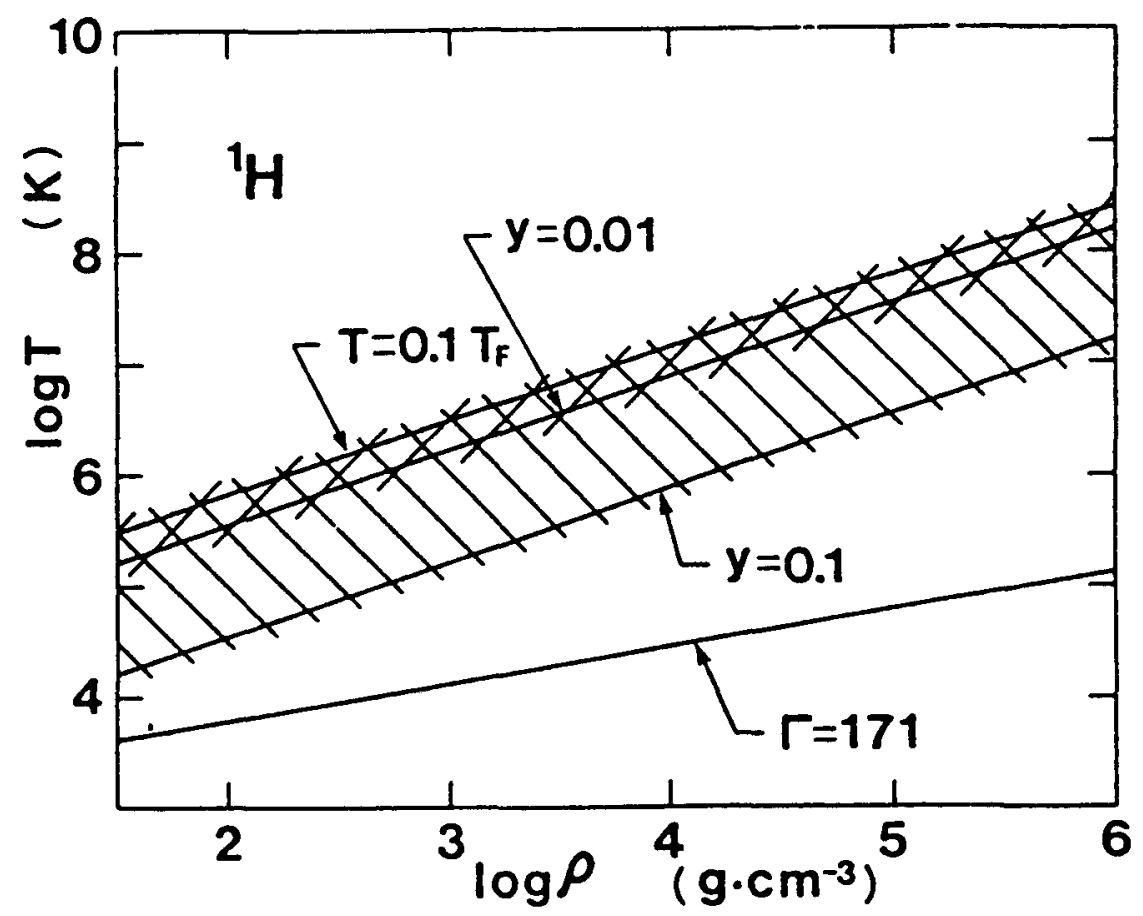

Fig. 18.1 The density-temperature diagram for the ${ }^{1} \mathrm{H}$ matter.

\& Itoh (1984) is based on the lowest-order expansions with respect to the small values of $y$. Thus the method completely breaks down for $y \geq 1$.

The expressions for the electrical conductivity $\sigma$ and the thermal conductivity $\kappa$ in the liquid metal phase are

$$
\begin{aligned}
& \sigma= 8.693 \times 10^{21} \frac{\rho_{6}}{A} \frac{1}{\left[1+1.018(Z / A)^{2 / 3} \rho_{6}^{2 / 3}\right]<S>} \mathrm{s}^{-1} \\
& \kappa= 2.363 \times 10^{17} \frac{\rho_{6} T_{8}}{A} \frac{1}{\left[1+1.018(Z / A)^{2 / 3} \rho_{6}^{2 / 3}\right]<S>} \\
& \operatorname{ergscm}^{-1} \mathrm{~s}^{-1} \mathrm{~K}^{-1}, \\
&<S>=\int_{0}^{1} d\left(\frac{k}{2 k_{F}}\right)\left(\frac{k}{2 k_{F}}\right)^{3} \frac{S\left(k / 2 k_{F}\right)}{\left[\left(k / 2 k_{F}\right)^{2} \epsilon\left(k / 2 k_{F}, 0\right)\right]^{2}} \\
&-\frac{1.018(Z / A)^{2 / 3} \rho_{6}^{2 / 3}}{1+1.018(Z / A)^{2 / 3} \rho_{6}^{2 / 3}}
\end{aligned}
$$




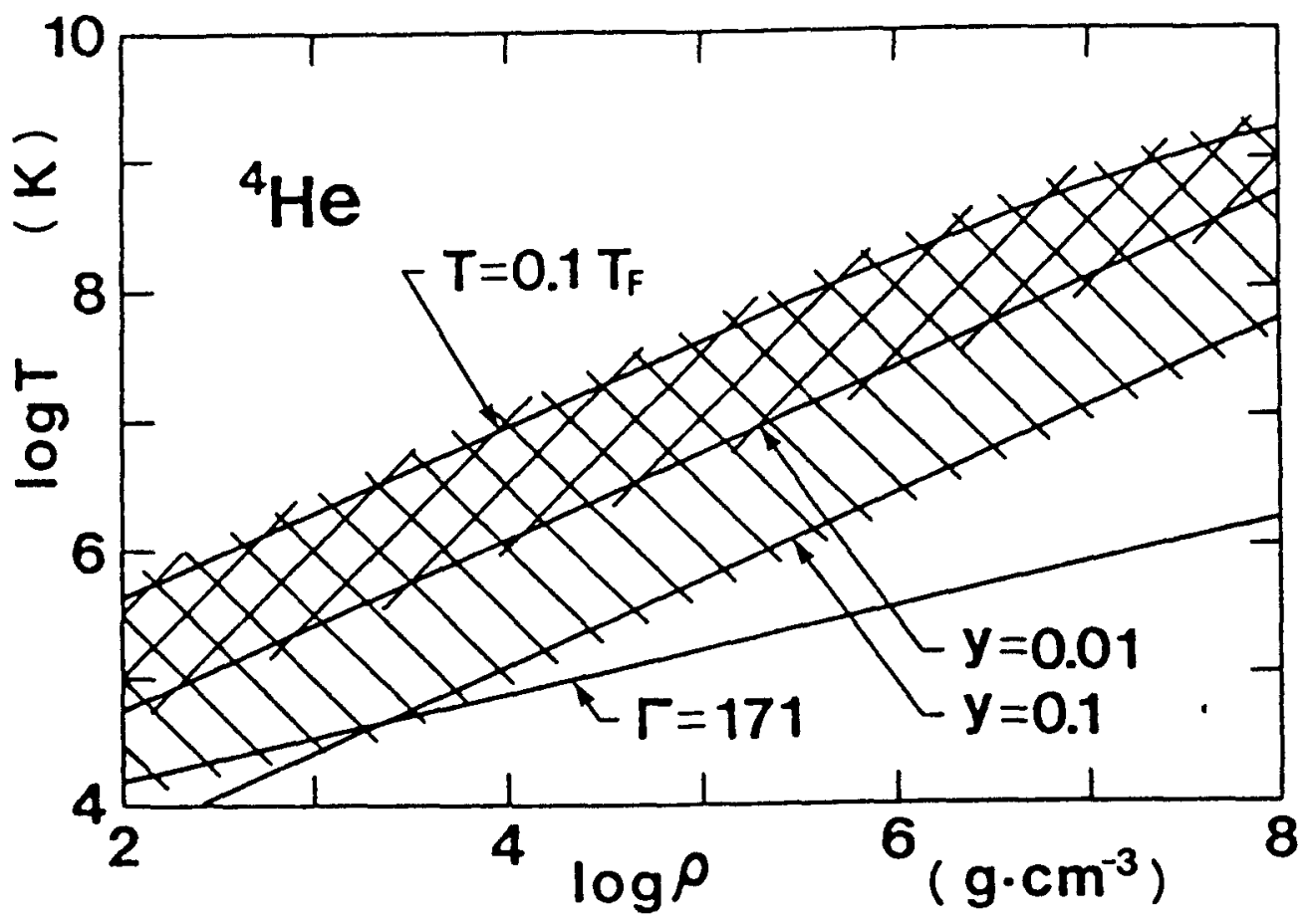

Fig. 18.2 The density-temperature diagram for the ${ }^{4} \mathrm{He}$ matter.

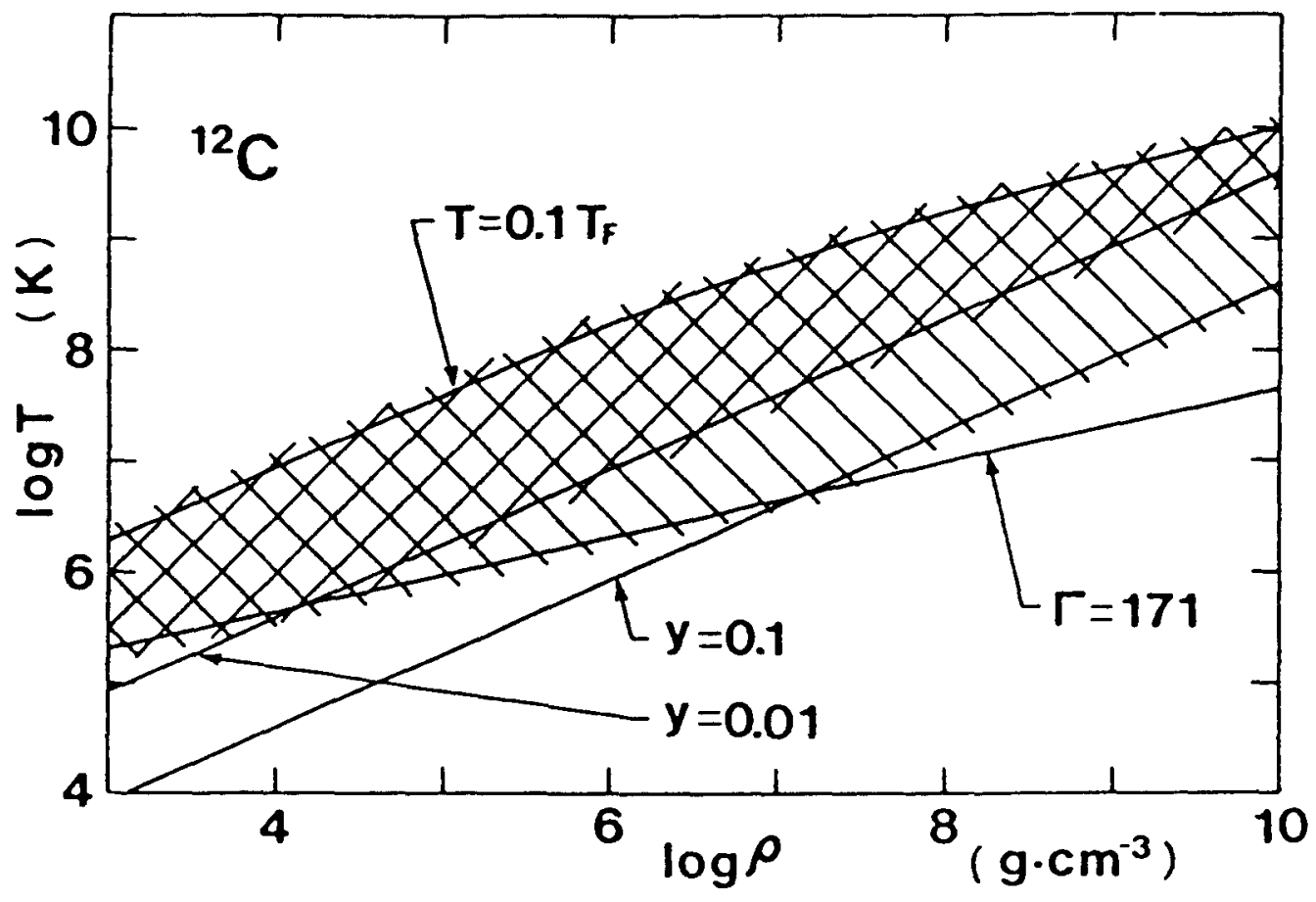

Fig. 18.3 The density-temperature diagram for the ${ }^{12} \mathrm{C}$ matter. 


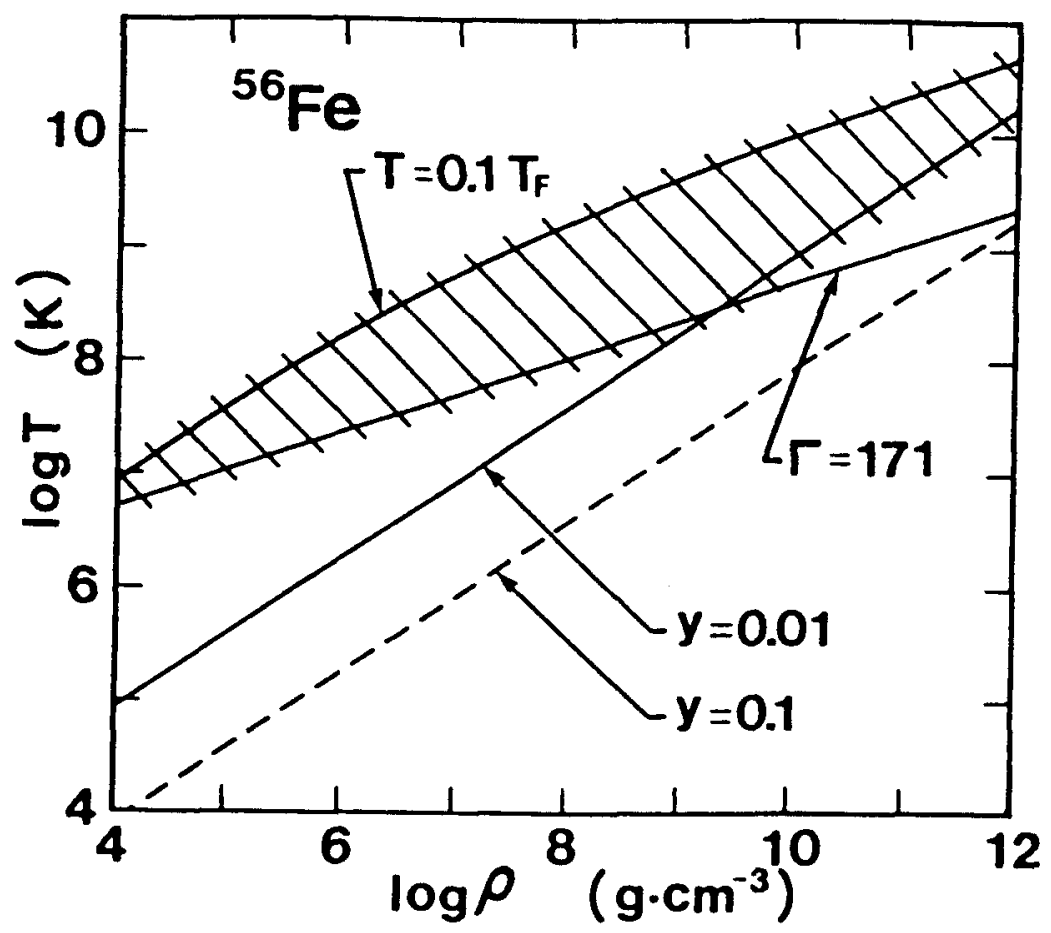

Fig. 18.4 The density-temperature diagram for the ${ }^{56} \mathrm{Fe}$ matter.

$$
\begin{aligned}
& \times \int_{0}^{1} d\left(\frac{k}{2 k_{F}}\right)\left(\frac{k}{2 k_{F}}\right)^{5} \frac{S\left(k / 2 k_{F}\right)}{\left[\left(k / 2 k_{F}\right)^{2} \epsilon\left(k / 2 k_{F}, 0\right)\right]^{2}} \\
& \left.\equiv<S_{-1}>-\frac{1.018(Z / A)^{2 / 3} \rho_{6}^{2 / 3}}{1+1.018(Z / A)^{2 / 3} \rho_{6}^{2 / 3}}<S_{+1}\right\rangle,
\end{aligned}
$$

where $\hbar k$ is the momentum transferred from the ionic system to the electron, $S\left(k / 2 k_{F}\right)$ is the ionic liquid structure factor, and $\epsilon\left(k / 2 k_{F}, 0\right)$ is the static dielectric screening function due to the degenerate electrons. The first term in equation (8) corresponds to the ordinary Coulomb logarithmic term, and the second term is a relativistic correction term.

In Itoh et al. (1983) explicit calculations have been carried out for $Z=$ $1,2,6,8,10,12,14,16,20,26$, and asymptotic formulae have been presented for $Z \geq 27$. Some of the results are shown in Figures 5-8.

The difference between the results of Yakovlev and Urpin (1981) and those of Itoh et al. (1983) is the following: Itoh et al. (1983) carried out the integrations in equation (8) by using the IHNC structure factor of the classical one-component plasma calculated by Iyetomi \& Ichimaru (1982) and Jancovici's (1962) relativistic dielectric function for the degenerate electrons, whereas Yakovlev \& Urpin (1981) used an approximate form of the srtuc- 


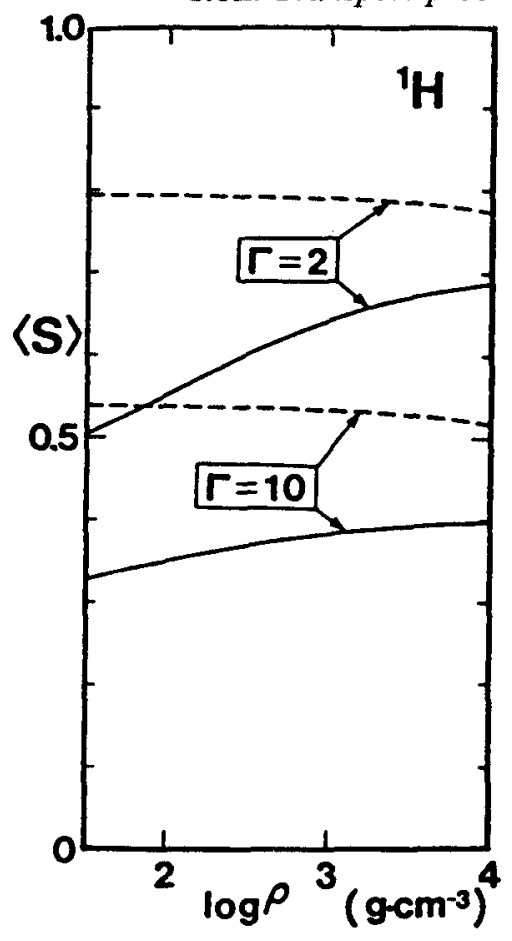

Fig. 18.5 Comparison of Yakovlev and Urpin's results (dashed curves) with those of Itoh et al. (solid curves) for the ${ }^{1} \mathrm{H}$ matter.

ture factor and neglected the screening due to the degenerate electrons by setting $\epsilon\left(k / 2 k_{F}, 0\right)=1$. Yakovlev and Urpin's (1981) approximations are quite satisfactory at high densities as can be seen from Figures $5-8$, but bring about significant deviations from the correct results at low densities where the screening due to the degenerate electrons is appreciable. Accurate analytical fitting formulae which summarize the numerical results have been presented by Itoh et al. (1983)

\subsection{Electrical and Thermal Conductivities in the Crystalline Lattice Phase}

In this section we review the calculations of the electrical and thermal conductivities of dense matter in the crystalline latttice phase $\left(\Gamma \geq \Gamma_{m}=180\right)$ following the papers of Itoh et al. (1984a) and Itoh,Hayashi,\&Kohyama (1993).

The relativistic extension of the expressions for the electrical and thermal conductivities due to degenerate electrons has been given by Flowers \& Itoh (1976). The electrical conductivity $\sigma$ and thermal conductivity $\kappa$ are related to the effective electron collision frequencies $\nu_{\sigma}$ and $\nu_{\kappa}$ by 


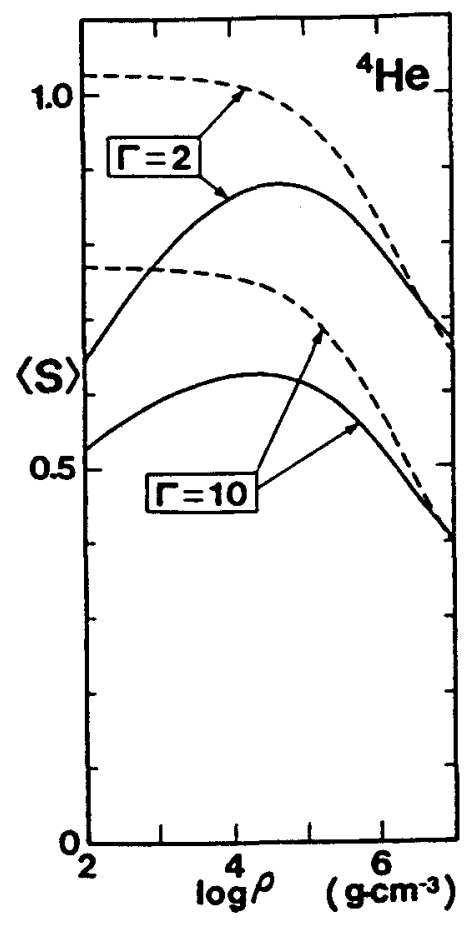

Fig. 18.6 Comparison of Yakovlev and Urpin's results (dashed curves) with those of Itoh et al. (solid curves) for the ${ }^{4} \mathrm{He}$ matter.

$$
\begin{aligned}
\sigma= & \frac{e^{2} n_{e}}{m^{*} \nu_{\sigma}}=1.525 \times 10^{20} \frac{Z}{A} \rho_{6} \\
& \times\left[1+1.018\left(\frac{Z}{A} \rho_{6}\right)^{2 / 3}\right]^{-1 / 2} \frac{10^{18} \mathrm{~s}^{-1} \mathrm{~s}^{-1}}{\nu_{\sigma}}, \\
\kappa= & \frac{\pi^{2} k_{\mathrm{B}}^{2} T n_{e}}{3 m^{*} \nu_{\kappa}}=4.146 \times 10^{15} \frac{Z}{A} \rho_{6}\left[1+1.018\left(\frac{Z}{A} \rho_{6}\right)^{2 / 3}\right]^{-1 / 2} \\
& \times T_{8} \frac{10^{18} \mathrm{~s}^{-1}}{\nu_{\kappa}} \text { ergs } \mathrm{cm}^{-1} \mathrm{~s}^{-1} \mathrm{~K}^{-1}
\end{aligned}
$$

where $n_{e}$ is the number density of eletrons and $m^{*}$ is the relativistic effective mass of an electron at the Fermi surface. In this section we are interested in the scattering of electrons by phonons. The collision frequencies $\nu_{\sigma}$ and $\nu_{\kappa}$ due to one-phonon processes can be calculated by the variational method (Flowers \& Itoh 1976; Yakovlev \& Urpin 1981; Raikh \& Yakovlev 1982) as 


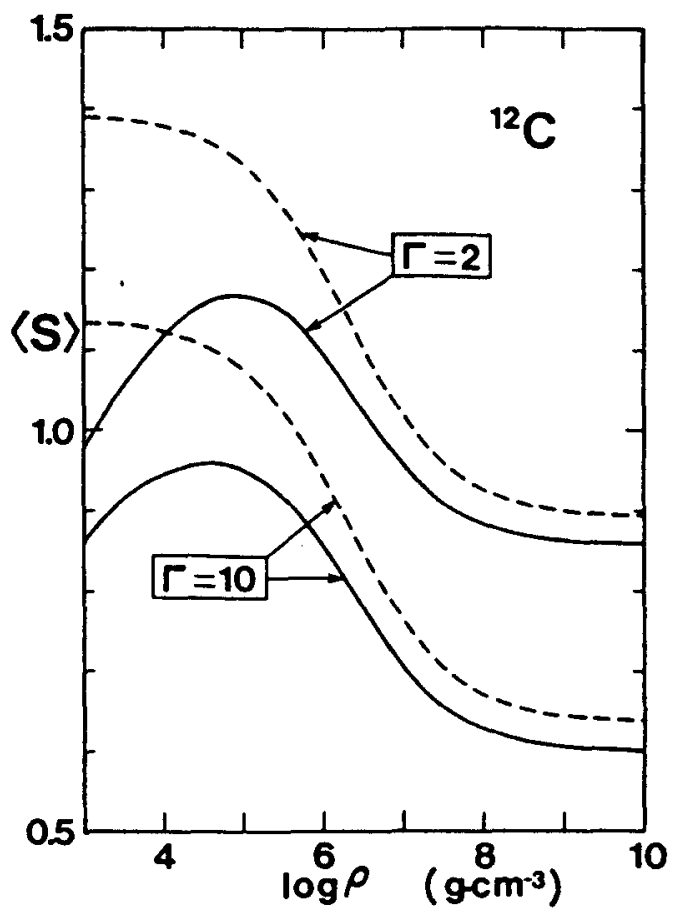

Fig. 18.7 Comparison of Yakovlev and Urpin's results (dashed curves) with those of Itoh et al. (solid curves) for the ${ }^{12} \mathrm{C}$ matter.

$$
\begin{aligned}
\nu_{\sigma, \kappa} & =\frac{e^{2}}{\hbar v_{\mathrm{F}}} \frac{k_{\mathrm{B}} T}{\hbar} F_{\sigma, \kappa} \\
& =9.554 \times 10^{16} T_{\mathrm{B}}\left\{1+\frac{1}{1.018\left[(Z / A) \rho_{6}\right]^{2 / 3}}\right\}^{1 / 2} F_{\sigma, \kappa} \mathrm{s}^{-1}, \\
F_{\sigma, \kappa} & =\frac{2 \gamma^{2}}{S^{2}} \int \frac{d S d S^{\prime}}{k^{4}|\varepsilon(k, 0)|^{2}}\left[1-\left(\frac{\beta k}{2 k_{\mathrm{F}}}\right)^{2}\right] e^{-2 W(k)} \\
& \times|f(k)|^{2} \sum_{s=1}^{3}\left[\mathrm{k} \cdot \hat{\epsilon}_{\mathrm{s}}(\mathrm{p})\right]^{2}\left(e^{z_{s}}-1\right)^{-2} e^{z_{\bullet}} g_{\sigma, \kappa} .
\end{aligned}
$$

In the above the integral is over the areas of the Fermi surface, $\mathbf{k}$ is the momentum transfer, $\hat{\epsilon}_{\bullet}(\mathbf{p})$ is the polarization unit vector of a phonon with momentum $\mathbf{p}$ and polarization $\mathrm{s}$, and

$$
\gamma \equiv \frac{\hbar \omega_{p}}{k_{\mathrm{B}} T}=7.832 \times 10^{-2} \frac{Z}{A} \frac{\rho_{6}^{1 / 2}}{T_{8}}=0.3443 \frac{\rho_{6}^{1 / 6}}{A^{2 / 3} Z} \Gamma,
$$




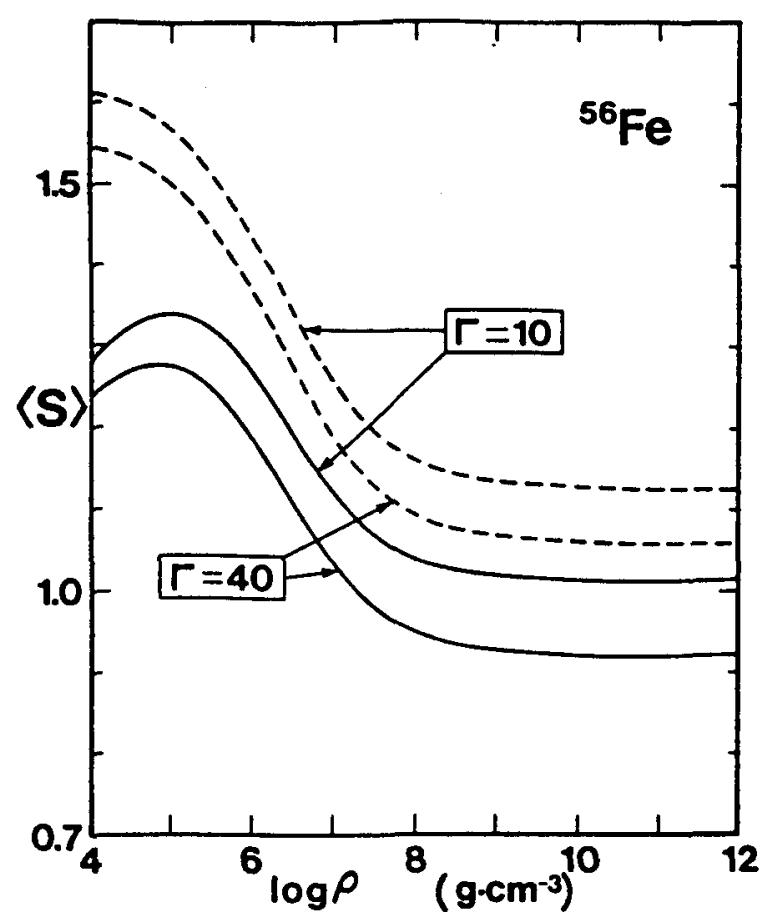

Fig. 18.8 Comparison of Yakovlev and Urpin's results (dashed curves) with those of Itoh et al. (solid curves) for the ${ }^{56} \mathrm{Fe}$ matter.

$$
\begin{aligned}
& \beta \equiv \frac{\hbar k_{\mathrm{F}} c}{E_{\mathrm{F}}}=\left\{1+\frac{1}{1.018\left[(Z / A) \rho_{6}\right]^{2 / 3}}\right\}^{-1 / 2}, \\
& z_{s} \equiv \frac{\hbar \omega_{s}(\mathrm{p})}{k_{\mathrm{B}} T} \\
& g_{\sigma}=k^{2} \\
& g_{\kappa}=k^{2}-\frac{k^{2} z_{s}^{2}}{2 \pi^{2}}+\frac{3 k_{\mathrm{F}}^{2} z_{s}^{2}}{\pi^{2}},
\end{aligned}
$$

$\omega_{p}$ being the ionic plasma frequency. The momentum conservation requires $\mathbf{k}= \pm \mathbf{p}+\mathbf{K}$, where $\mathbf{K}$ is the reciprocal-lattice vector for the Brillouin zone to which $\mathbf{k}$ is confined.

In equation (12) we have included the dielectric screening function due to relativistically degenerate electrons $\varepsilon(k, 0)$, the Debye - Waller factor $e^{-2 W(k)}$, and the atomic form factor $f(k)$. Yakovlev \& Urpin (1981) and Raikh \& Yakovlev (1982) have used the Thomas - Fermi screening and set $e^{-2 W(k)}=1, f(k)=1$. 


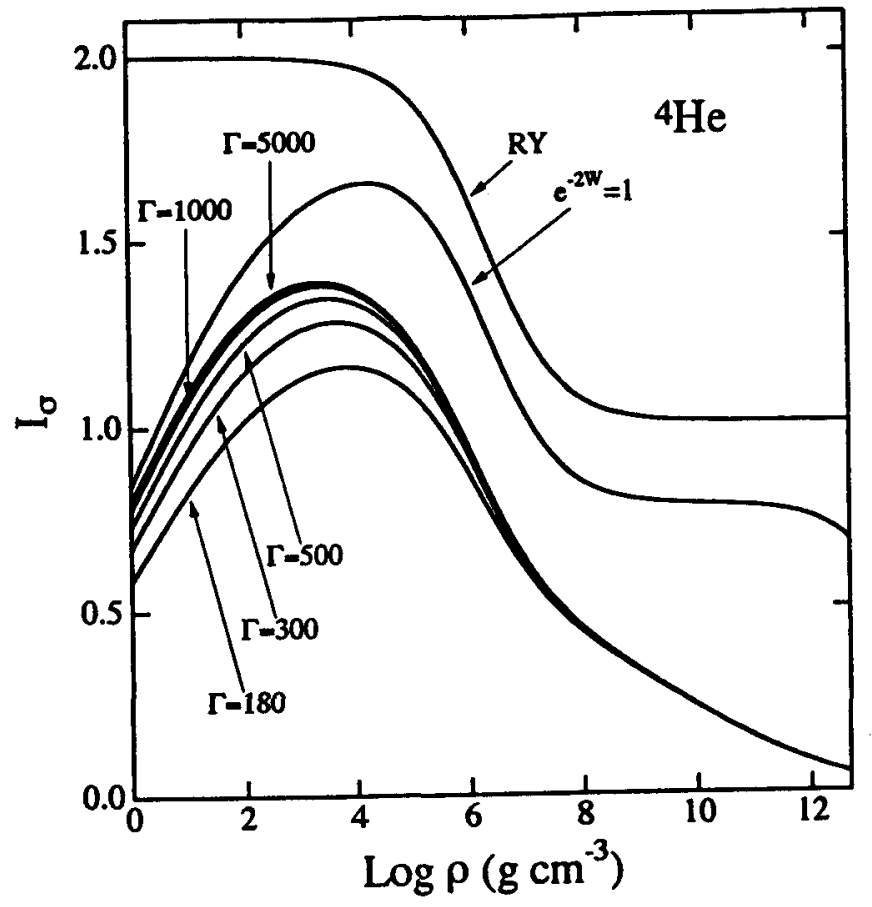

Fig. 18.9 $I_{\sigma}$ for the ${ }^{4} \mathrm{He}$ matter. RY stands for the results of Raikh \& Yakovlev (1982).

The static longitudinal dielectric function due to relativistically degenerate electrons (Jancovici 1962) is given by

$$
\begin{aligned}
& \varepsilon(q, 0)=1+\left(\frac{2}{3 \pi^{2}}\right)^{2 / 3} \frac{r_{s}}{q^{2}}\left[\frac{2}{3}\left(1+x^{2}\right)^{1 / 2}\right. \\
& \quad-\frac{2 q^{2} x}{3} \sinh ^{-1} x+\left(1+x^{2}\right)^{1 / 2} \frac{x^{2}+1-3 q^{2} x^{2}}{6 q x^{2}} \ln \left|\frac{1+q}{1-q}\right| \\
& \left.\quad+\frac{2 q^{2} x^{2}-1}{6 q x^{2}}\left(1+q^{2} x^{2}\right)^{1 / 2} \ln \left|\frac{q\left(1+x^{2}\right)^{1 / 2}+\left(1+q^{2} x^{2}\right)^{1 / 2}}{q\left(1+x^{2}\right)^{1 / 2}-\left(1+q^{2} x^{2}\right)^{1 / 2}}\right|\right], \\
& q=\frac{k}{2 k_{\mathrm{F}}}, \\
& x=\frac{\hbar k_{\mathrm{F}}}{m_{e} c}=\frac{1}{137.0}\left(\frac{9 \pi}{4}\right)^{1 / 3} r_{s}^{-1}, \\
& r_{s}=1.388 \times 10^{-2}\left(\frac{A}{Z \rho_{6}}\right)^{1 / 3} .
\end{aligned}
$$




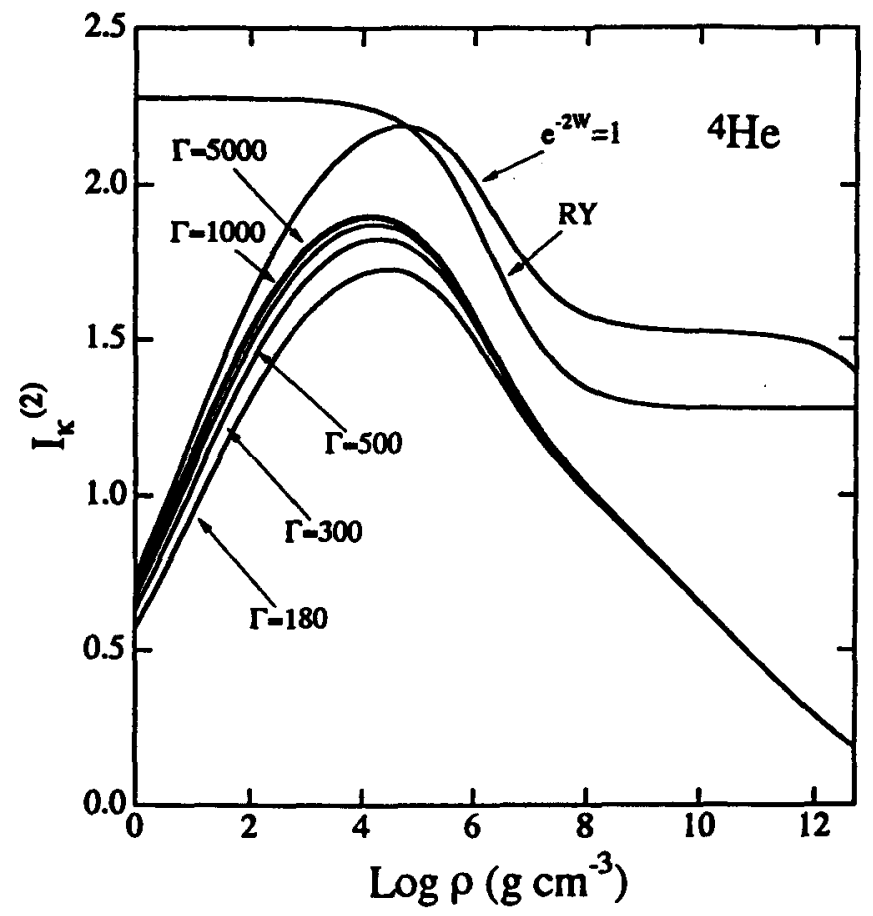

Fig. $18.10 I_{\kappa}^{(2)}$ for the ${ }^{4} \mathrm{He}$ matter.

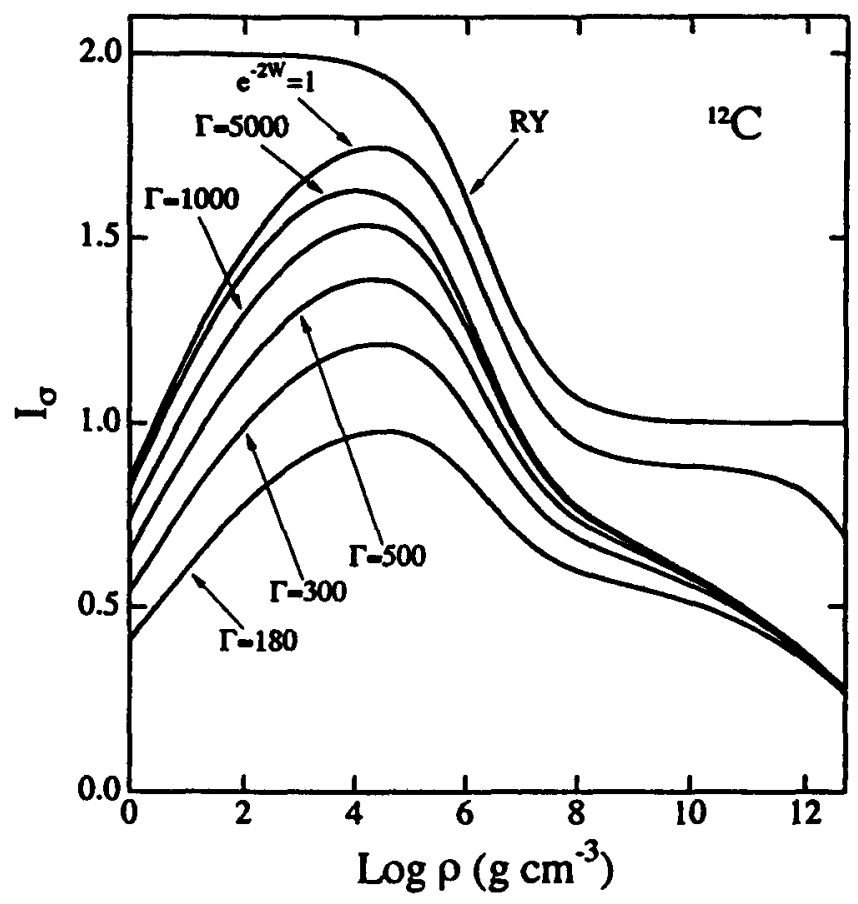

Fig. 18.11 $I_{\sigma}$ for the ${ }^{12} \mathrm{C}$ matter. 


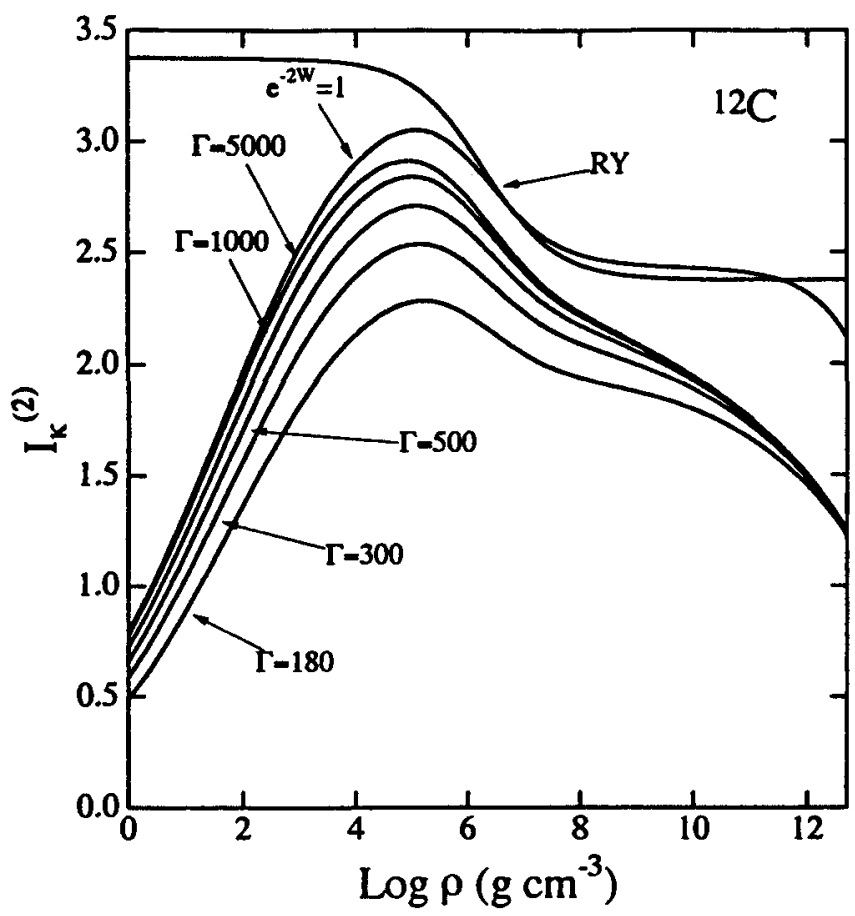

Fig. 18.12 $I_{\kappa}^{(2)}$ for the ${ }^{12} \mathrm{C}$ matter.

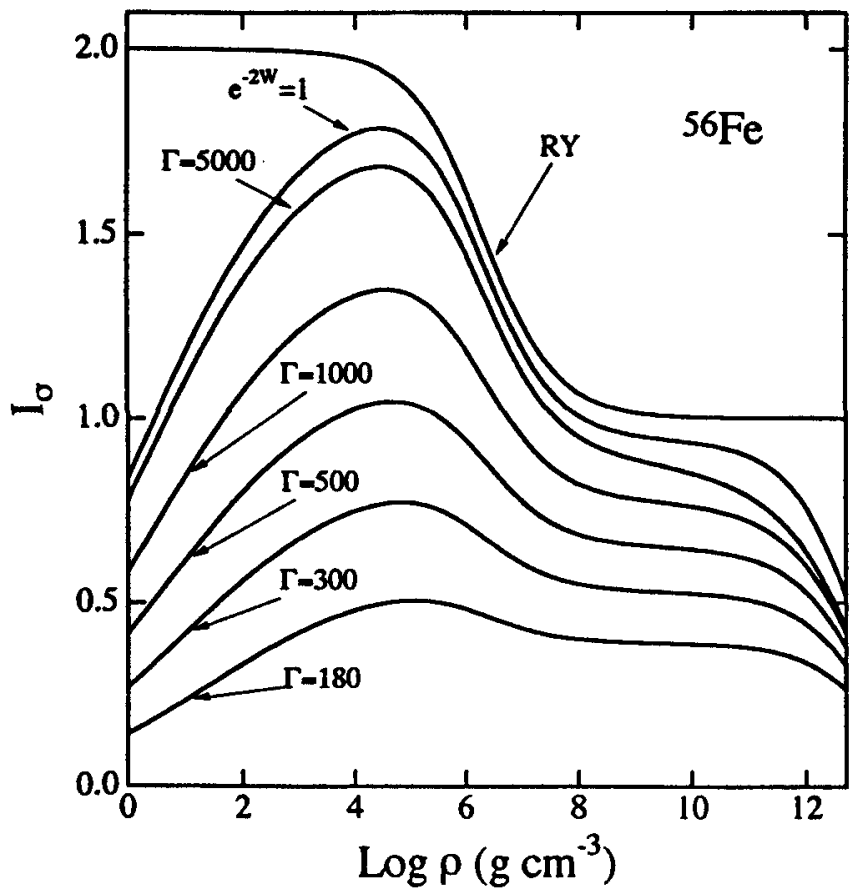

Fig. 18.13 $I_{\sigma}$ for the ${ }^{56} \mathrm{Fe}$ matter. 


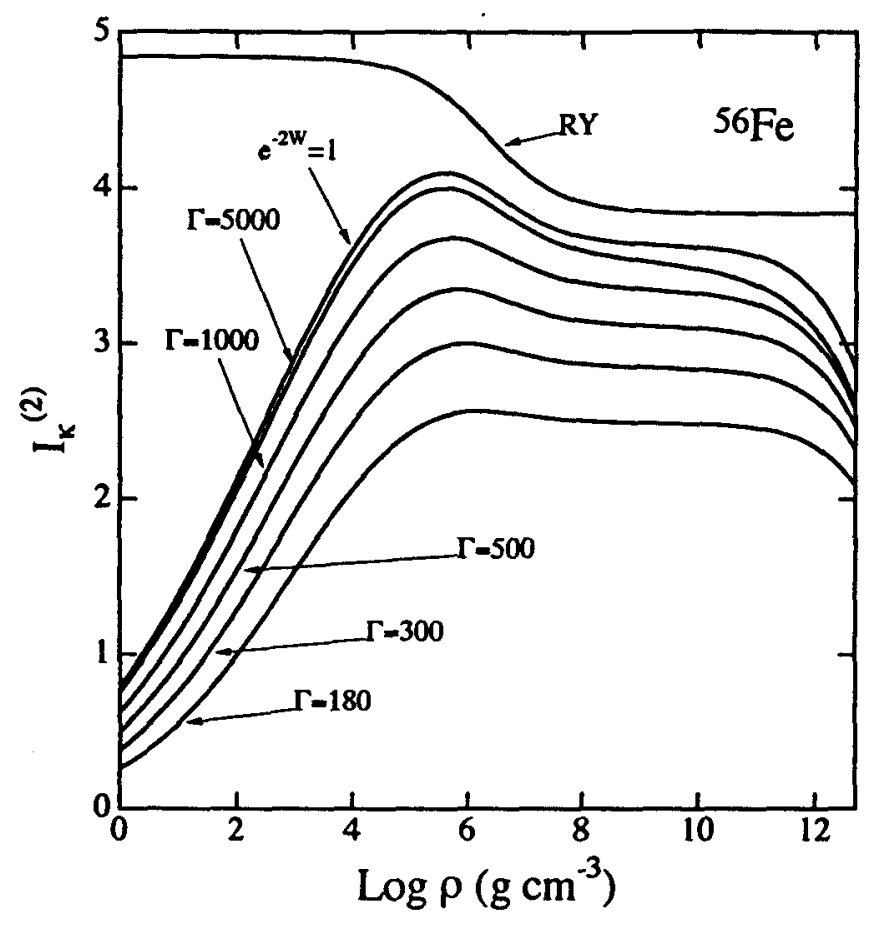

Fig. $18.14 I_{\kappa}^{(2)}$ for the ${ }^{56} \mathrm{Fe}$ matter.

The electron Fermi wavenumber is expressed as

$$
k_{\mathrm{F}}=2.613 \times 10^{10}\left(\frac{Z}{A} \rho_{6}\right)^{1 / 3} \mathrm{~cm}^{-1}
$$

The Debye - Waller factor is written as

$$
2 W(k)=\sum_{k^{\prime} s^{\prime}} \frac{\hbar\left[\mathbf{k} \cdot \hat{\epsilon}_{s^{\prime}}\left(\mathbf{k}^{\prime}\right)\right]^{2}}{2 M N \omega_{s^{\prime}}\left(\mathbf{k}^{\prime}\right)} \operatorname{coth}\left(\frac{\hbar \omega_{s^{\prime}}\left(\mathbf{k}^{\prime}\right)}{2 k_{\mathrm{B}} T}\right)
$$

where $M$ is the mass of an ion and $N$ is the number of ions in the lattice. Itoh et al. (1984b) have obtained the practical formula for the Debye Waller factor for the pure Coulomb bcc lattice which satisfies the frequency moment sum rules for both $k_{\mathrm{B}} T \gg \hbar \omega_{p}$ and $k_{\mathrm{B}} T \ll \hbar \omega_{p}$ (Coldwell-Horsfall \& Maradudin 1960; Pollock \& Hansen 1973). The practical formula for the Debye - Waller factor reads as

$$
2 W(k)=241.5 q^{2} \alpha \gamma^{-3} \int_{0}^{0.4306 \gamma}\left(\frac{1}{e^{z}-1}+\frac{1}{2}\right) z d z
$$


408

$$
\begin{aligned}
\alpha \equiv & \frac{\hbar^{2} k_{\mathrm{F}}^{2}}{2 M k_{\mathrm{B}} T}=1.656 \times 10^{-2} \frac{1}{A T_{8}}\left(\frac{Z}{A} \rho_{6}\right)^{2 / 3} \\
& =0.2114 \frac{\rho_{6}^{1 / 6}}{A^{2 / 3} Z^{1 / 3}} \gamma .
\end{aligned}
$$

The practical approximations of the Debye function that appears in equation (20) are written as follows:

$$
\begin{aligned}
& \int_{0}^{x}\left(\frac{2}{e^{z}-1}+\frac{1}{2}\right) z d z \\
& \approx x\left(1+\frac{1}{6 \cdot 3 !} x^{2}-\frac{1}{30 \cdot 5 !} x^{4}+\frac{1}{42 \cdot 7 !} x^{6}-\frac{1}{30 \cdot 9 !} x^{8}+\frac{5}{66 \cdot 11 !} x^{10}\right) \\
& \quad \text { for } x<2.5 \\
& \int_{0}^{x}\left(\frac{1}{e^{z}-1}+\frac{1}{2}\right) z d z \approx \frac{\pi^{2}}{6}+\frac{1}{4} x^{2}-\sum_{n=1}^{5} \frac{1}{n}\left(x+\frac{1}{n}\right) e^{-n x} \\
& \quad \text { for } x>2.5 .
\end{aligned}
$$

The errors of formulae (26) and (27) at $x=2.5$ are both smaller than $10^{-5}$.

When the de Broglie wavelength of the electron becomes short enough to be comparable to the nuclear radius, we have to take into account the finite nuclear size corrections for the Coulomb scattering. This is done by multiplying the matrix element by the atomic form factor

$$
f(q)=-3 \frac{\left(2 k_{\mathrm{F}} r_{c} q\right) \cos \left(2 k_{\mathrm{F}} r_{c} q\right)-\sin \left(2 k_{\mathrm{F}} r_{c} q\right)}{\left(2 k_{\mathrm{F}} r_{c} q\right)^{3}} .
$$

The charge radius of the nucleus is represented by

$$
r_{c}=1.15 \times 10^{-13} A^{1 / 3} \mathrm{~cm} .
$$

The phonon spectra are modified by the screening due to electrons. The longitudinal optical phonon turns into an acoustic phonon in the longwavelength limit, whereas the original transverse acoustic phonons are little affected by the electron screening (Pollock \& Hansen 1973). Because the low-frequency transverse phonons play dominant roles in the resistivity of dense stellar matter, we neglect the effects of the electron screening on 
the phonon spectra and use the frequency moment sum rules for the pure Coulomb lattice.

As we consider the case in which the Fermi sphere is much larger than the Debye sphere, $\left(k_{\mathrm{F}} / k_{\mathrm{D}}\right)^{3}=Z / 2 \gg 1$, Umklapp processes contribute to the scattering dominantly, and the vector $k$ in equation (12) most probably falls in a Brillouin zone distant from the first zone. When we perform an integration within a single distant zone corresponding to the reciprocal lattice vector $K$, we can make an approximation $k=K$ in the integrand and carry out an integration over $\mathbf{p}$ within the first zone only.

Here we follow the semianalytical approach adopted by Yakovlev \& Urpin (1981) and also by Raikh \& Yakovlev (1982). We write

$$
\begin{aligned}
& \sum_{s=1}^{3}\left[\mathbf{k} \cdot \hat{\epsilon}_{s}(\mathbf{p})\right]^{2} z_{s}^{n}\left(e^{z_{s}}-1\right)^{-2} e^{z_{\iota}} \approx \frac{\pi^{n} k^{2}}{\gamma^{2}} G^{(n)}(\gamma), \\
& G^{(n)}(\gamma)=\frac{\gamma^{2}}{3 V_{\mathrm{B}} \pi^{n}} \sum_{s=1}^{3} \int d \mathbf{p} z_{s}^{n}\left(e^{z_{s}}-1\right)^{-2} e^{z_{\iota}},
\end{aligned}
$$

where $n=0$ or 2 , and integration is carried out over the first Brillouin zone, whose volume is $V_{\mathrm{B}}$. By the use of this approximation $F_{\sigma}$ and $F_{\kappa}$ in equation (12) are expressed as

$$
\begin{aligned}
& F_{\sigma}=I_{\sigma} G^{(0)}(\gamma) \\
& F_{\kappa}=I_{\sigma} G^{(0)}(\gamma)+I_{\kappa}^{(2)} G^{(2)}(\gamma), \\
& I_{\sigma}=\int_{-1}^{\mu_{\max }} d \mu \frac{e^{-2 W(q)}|f(q)|^{2}}{|\varepsilon(q, 0)|^{2}}\left(1-\beta^{2} q^{2}\right), \\
& I_{\kappa}^{(2)}=\int_{-1}^{\mu_{\max }} d \mu \frac{e^{-2 W(q)}|f(q)|^{2}}{q^{2}|\varepsilon(q, 0)|^{2}}\left(1-\beta^{2} q^{2}\right)\left(-\frac{1}{2} q^{2}+\frac{3}{4}\right), \\
& q=\left(\frac{1-\mu}{2}\right)^{1 / 2}, \\
& q_{\min }=\left(\frac{1-\mu_{\max }}{2}\right)^{1 / 2}, \\
& \mu_{\max }=1-0.3575 Z^{-2 / 3} .
\end{aligned}
$$


Here we have introduced a small momentum transfer cutoff $q_{\min }$ corresponding to the unavailability of Umklapp processes for $q<q_{\min }$. The contributions of the normal processes are very much smaller than those of the Umklapp processes. For the choise of $q_{\min }$ we have followed Raikh \& Yakovlev (1982). Yakovlev \& Urpin (1981) derived the asymptotic expressions of $G^{(0)}(\gamma)$ and $G^{(2)}(\gamma)$ for $\gamma \ll 1$ and $\gamma \gg 1$, and proposed the following analytic formulae for arbitrary $\gamma$, which fit the main terms of the asymptotic expressions:

$$
\begin{aligned}
& G^{(0)}(\gamma)=u_{-2}\left[1+\left(\frac{3 u_{-2} \gamma}{\pi^{2} c_{2}}\right)^{2}\right]^{-1 / 2} \\
& \approx 13.00\left(1+0.0174 \gamma^{2}\right)^{-1 / 2} \\
& G^{(2)}(\gamma)=\frac{\gamma^{2}}{\pi^{2}}\left[1+\left(\frac{15}{4 \pi^{4} c_{2}}\right)^{2 / 3} \gamma^{2}\right]^{-3 / 2} \\
& =\frac{\gamma^{2}}{\pi^{2}}\left(1+0.0118 \gamma^{2}\right)^{-3 / 2}
\end{aligned}
$$

where $u_{-2} \approx 13.00$ (Pollock \& Hansen 1973) and $c_{2}=29.98$ (ColdwellHorsfall \& Maradudin 1960) are the numerical constants that are characteristic of the phonon spectrum of the bcc Coulomb lattice. Raikh \& Yakovlev (1982) calculated $G^{(0)}(\gamma)$ and $G^{(2)}(\gamma)$ numerically with the exact spectrum of phonons for $\gamma<100$. It has been confirmed that the fitting formulae (39) and (40) have an accuracy better than $10 \%$ even at $\gamma \sim 1$.

We have carried out the numerical integrations of equations (34) and (35) for ${ }^{4} \mathrm{He},{ }^{12} \mathrm{C},{ }^{16} \mathrm{O},{ }^{20} \mathrm{Ne},{ }^{24} \mathrm{Mg},{ }^{28} \mathrm{Si},{ }^{32} \mathrm{~S},{ }^{40} \mathrm{Ca}$, and ${ }^{56} \mathrm{Fe}$. Some of the results are presented in Figures 1-6. For comparison we have also included the case where we have neglected the effects of the Debye - Waller factor and set $e^{-2 W}=1$. We also show the results of Raikh \& Yakovlev (1982), which are

$$
\begin{aligned}
& {\left[I_{\sigma}\right]_{\mathrm{RY}}=2-\beta^{2},} \\
& {\left[I_{\kappa}^{(2)}\right]_{\mathrm{RY}}=\ln Z-\beta^{2}+1.583 .}
\end{aligned}
$$

It is readily seen that the Debye - Waller factor reduces the resistivities (enhances the conductivities) by a factor of $2-4$ near the melting temperature. 


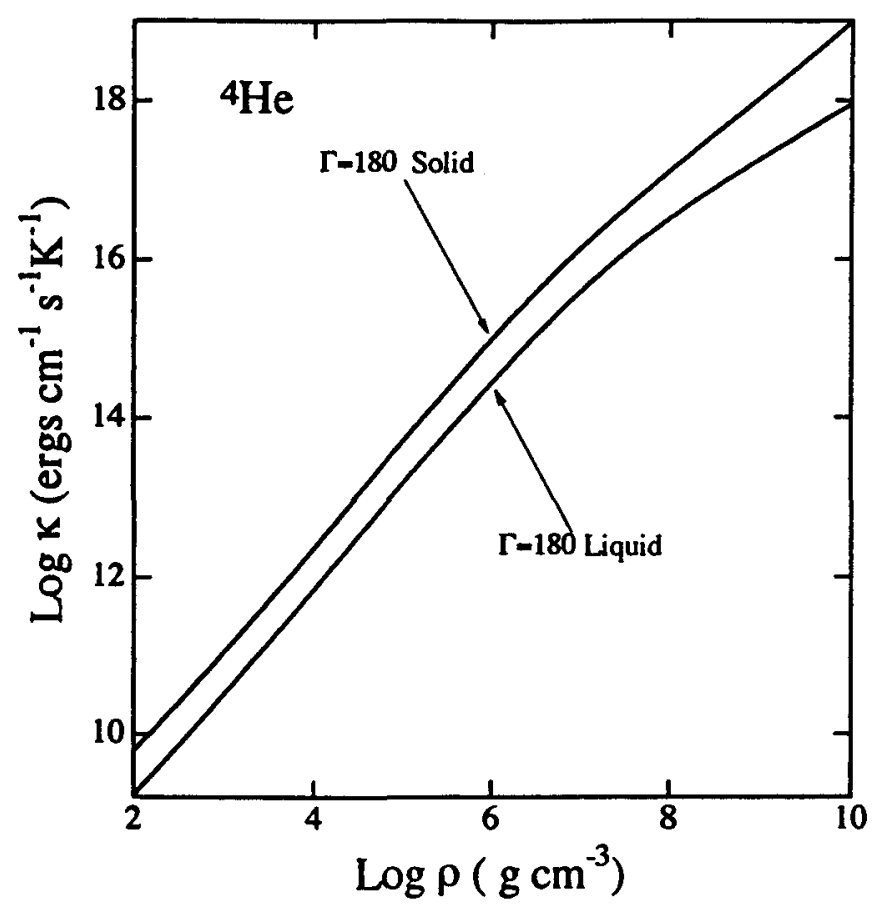

Fig. 18.15 Thermal conductivities of the ${ }^{4} \mathrm{He}$ matter at the crystallization point $\Gamma=180$.

In Figures $15-17$ we show the thermal conductivities at the crystallization point $\Gamma=180$ as functions of densities. Thermal conductivities in the crystalline lattice phase are the present results. Thermal conductivities in the liquid metal phase have been calculated by using the method of Itoh et al. (1983). It is found that the thermal conductivity in the crystalline lattice phase is generally higher than that in the liquid metal phase by a factor $2-4$ at the crystallization point $\Gamma=180$.

In Figure 18 we show the thermal conductivity of the "pure" ${ }^{12} \mathrm{C}$ matter as a function of the parameter $\Gamma$ for the densities $10^{4} \mathrm{~g} \mathrm{~cm}^{-3}, 10^{6} \mathrm{~g} \mathrm{~cm}^{-3}$, $10^{8} \mathrm{~g} \mathrm{~cm}^{-3}$, and $10^{10} \mathrm{~g} \mathrm{~cm}^{-3}$. The thermal conductivity in the crystalline lattice phase is the present result, and the thermal conductivity in the liquid metal phase has been calculated by using the method of Itoh et al. (1983). The end point of the curve in the crystalline phase corresponds to the condition

$$
\gamma^{-1} \geq Z^{1 / 3} \frac{e^{2}}{\hbar \nu_{F}}=\left\{1+\frac{1}{1.018\left[(Z / A) \rho_{6}\right]^{2 / 3}}\right\}^{1 / 2}
$$




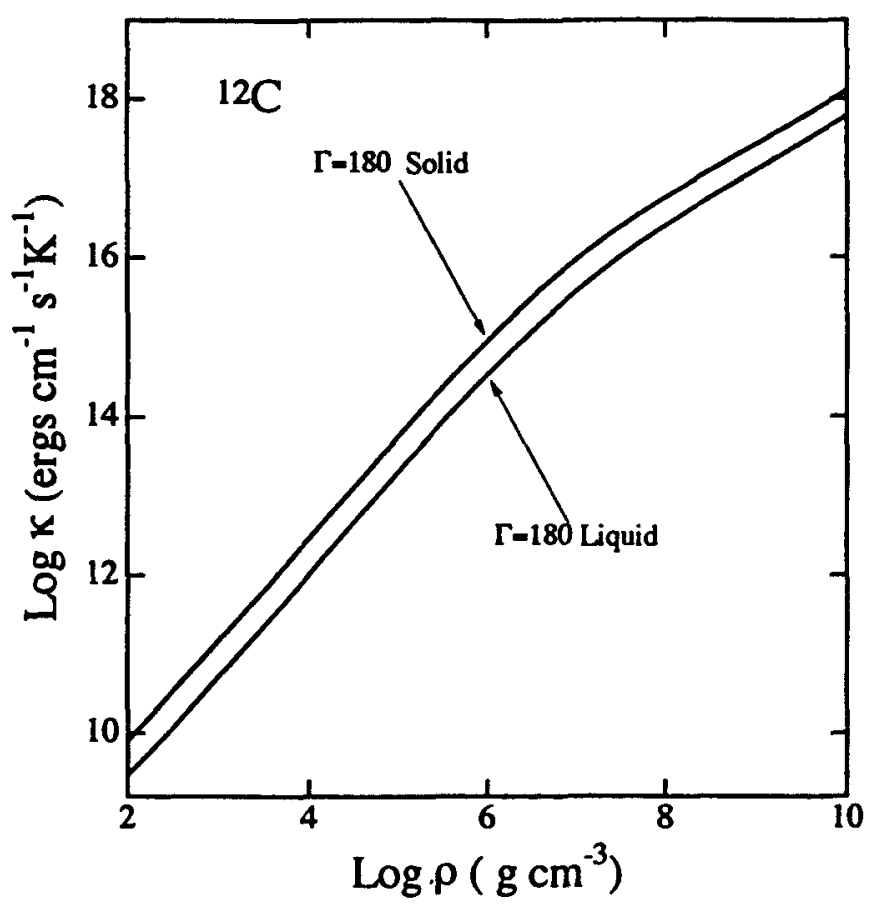

Fig. 18.16 Thermal conductivities of the ${ }^{12} \mathrm{C}$ matter at the crystallization point $\Gamma=180$.

which must hold in order that the Umklapp processes remain effective ( Yakovlev \& Urpin 1981; Raikh \& Yakovlev 1982).

We emphasize that in practice impurity scattering dominates over phonon scattering at low enough temperatures (Itoh \& Kohyama 1993). Therefore, the thermal conductivity in the crystalline phase presented here is an "ideal" thermal conductivity. Itoh, Hayashi, \& Kohyama (1993) have presented accurate analytical fitting formulae which summarize the numerical results of the calculation.

\subsection{Impurity Scattering Contributions in the Crystalline Lattice Phase}

In this section we review the calculation of the impurity scattering contributions in the crystalline lattice phase following the paper of Itoh \& Kohyama (1993). In the crystalline lattice phase, the phonon scattering and the impurity scattering contribute to the electrical and thermal conductivities in the following way:

$$
\sigma_{\text {tot }}^{-1}=\sigma_{\text {phonon }}^{-1}+\sigma_{\text {impurity }}^{-1}
$$




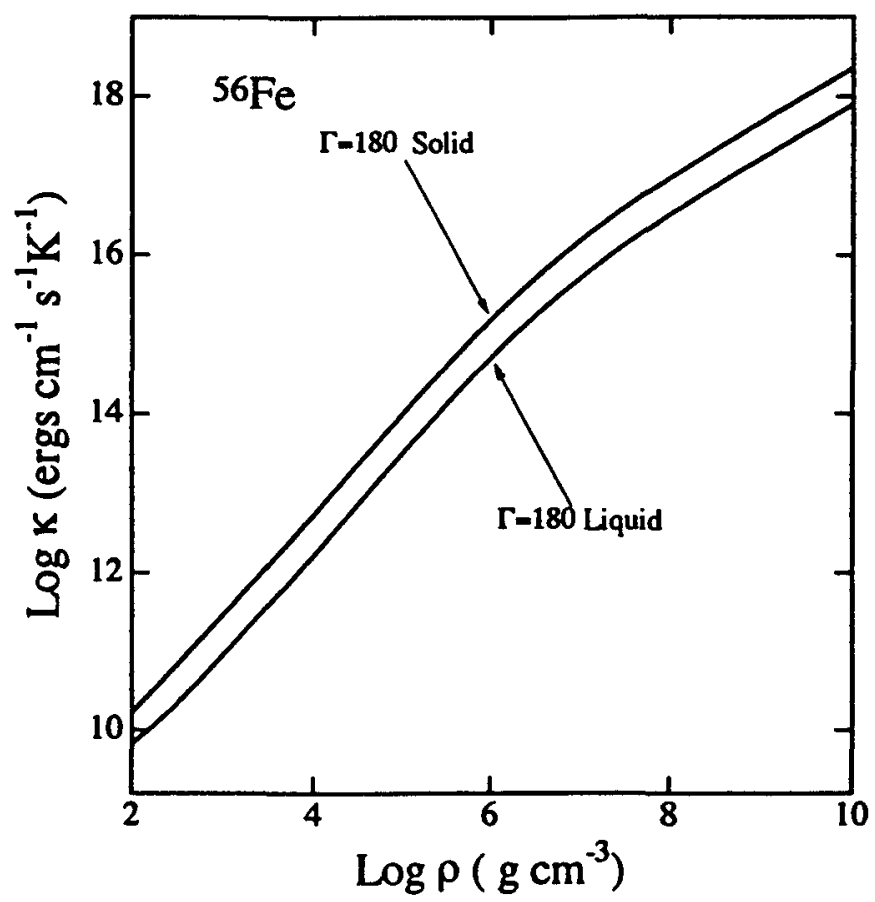

Fig. 18.17 Thermal conductivities of the ${ }^{56} \mathrm{Fe}$ matter at the crystallization point $\Gamma=180$.

$$
\kappa_{\text {tot }}^{-1}=\kappa_{\text {phonon }}^{-1}+\kappa_{\text {impurity }}^{-1} .
$$

The contributions of impurity scattering to electrical and thermal resistivities are thoroughly discussed in Ziman's (1960) authoritative book "Electrons and Phonons". We closely follow his ideas.

We suppose that there is a concentration $x$ of $\left(Z_{1}, A_{1}\right)$ ions and $(1-x)$ of $\left(Z_{2}, A_{2}\right)$ ions which forms a compositionally disordered lattice. This means that each lattice site is randomly occupied either by a $\left(Z_{1}, A_{1}\right)$ ion or a $\left(Z_{2}, A_{2}\right)$ ion. Thus we have

$$
x=\frac{n_{1}}{n_{1}+n_{2}}, 1-x=\frac{n_{2}}{n_{1}+n_{2}},
$$

where $n_{1}$ and $n_{2}$ are the number densities of the $\left(Z_{1}, A_{1}\right)$ and $\left(Z_{2}, A_{2}\right)$ ions, respectively.

We define an average charge and an average mass number

$$
\begin{aligned}
& <Z>=x Z_{1}+(1-x) Z_{2}, \\
& <A>=x A_{1}+(1-x) A_{2} .
\end{aligned}
$$

As the unperturbed state we take a regular lattice whose lattice sites are 


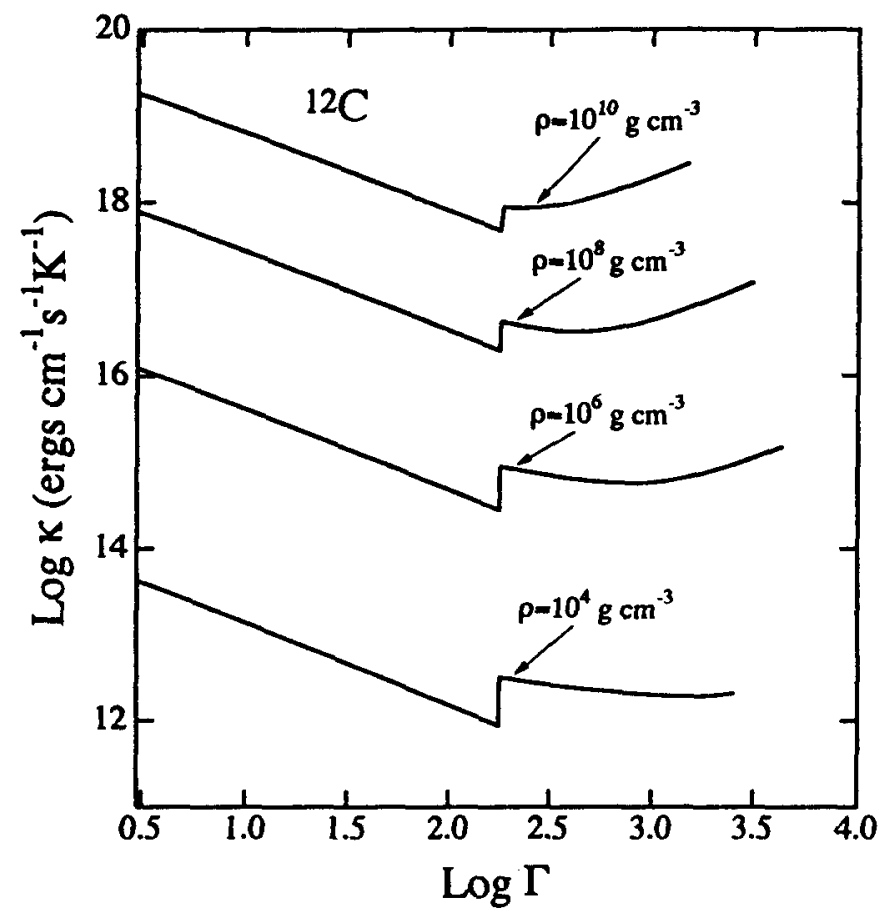

Fig. 18.18 Thermal conductivity of the "pure" ${ }^{12} \mathrm{C}$ matter as a function of the parameter $\Gamma$ for the densities $10^{4} \mathrm{gcm}^{-3}, 10^{6} \mathrm{gcm}^{-3}, 10^{8} \mathrm{gcm}^{-3}, 10^{10} \mathrm{gcm}^{-3}$.

occupied by $(<Z>,\langle A\rangle)$ ions. Then we suppose that impurity ions are added at lattice sites to make up the compositionally disordered lattice.

Thus at a site occupied by a $\left(Z_{1}, A_{1}\right)$ ion, the added impurity charge is

$$
Z_{1}-\langle Z\rangle=(1-x)\left(Z_{1}-Z_{2}\right) \equiv(1-x) \Delta Z \text {. }
$$

At a site occupied by a $\left(Z_{2}, A_{2}\right)$ ion, the added impurity charge is

$$
Z_{2}-\langle Z\rangle=-x \Delta Z \text {. }
$$

Next we consider these charge impurities as static, and calculate their contributions to electrical and thermal resistivities. This is exactly the same as that has been done by Itoh et al. (1983) when they calculated the electrical and thermal conductivities of dense matter in the liquid metal phase.

We consider the case that the atoms are completely pressure-ionized. We further restrict ourselves to the density-temperature region in which electrons are strongly degenerate. This condition is expressed as

$$
\begin{aligned}
T \ll & T_{F}=5.930 \times 10^{9} \\
& \times\left[\left[1+1.018\left(\frac{Z_{1}}{A_{1}} X_{1}+\frac{Z_{2}}{A_{2}} X_{2}\right)^{2 / 3} \rho_{6}^{2 / 3}\right]^{1 / 2}-1\right] \quad \mathrm{K},
\end{aligned}
$$


where $T_{F}$ is the electron Fermi temperature, $X_{1}$ and $X_{2}$ are the mass fractions $\left(X_{1}+X_{2}=1\right)$ of the ions $\left(Z_{1}, A_{1}\right)$ and $\left(Z_{2}, A_{2}\right)$, and $\rho_{6}$ is the mass density in units of $10^{6} \mathrm{gcm}^{-3}$. For the ionic system we consider the case that it is in the crystalline lattice state. The latest criterion corresponding to this condition for the case of the one-component system is given by (Ogata \& Ichimaru 1987)

$$
\Gamma \equiv \frac{Z^{2} e^{2}}{a k_{B} T}=2.275 \times 10^{-1} \frac{Z^{2}}{T_{8}}\left(\frac{\rho_{6}}{A}\right)^{1 / 3} \geq 180
$$

where $a=\left[3 /\left(4 \pi n_{i}\right)\right]^{1 / 3}$ is the ion-sphere radius, and $T_{8}$ is the temperature in units of $10^{8} \mathrm{~K}$.

Extending the work of Itoh et al. (1983) to the case of charged impurities, and assuming that the correlations between the two kinds of impurity charges are the same, we obtain the expressions for the electrical conductivity $\sigma$ and the thermal conductivity $\kappa$ limited by impurity scattering:

$$
\begin{aligned}
\sigma= & 8.693 \times 10^{21} \rho_{6}\left(\frac{X_{1}}{A_{1}}+\frac{X_{2}}{A_{2}}\right) \\
\times & \frac{1}{\left[1+1.018\left(\frac{Z_{1}}{A_{1}} X_{1}+\frac{Z_{2}}{A_{2}} X_{2}\right)^{2 / 3} \rho_{6}^{2 / 3}\right]<S>} \\
& \times \frac{1}{x(1-x)}\left(\frac{<Z>}{\Delta Z}\right)^{2} \mathrm{~s}^{-1} \\
\kappa= & 2.363 \times 10^{17} T_{8} \rho_{6}\left(\frac{X_{1}}{A_{1}}+\frac{X_{2}}{A_{2}}\right) \\
\times & \frac{1}{\left[1+1.018\left(\frac{Z_{1}}{A_{1}} X_{1}+\frac{Z_{2}}{A_{2}} X_{2}\right)^{2 / 3} \rho_{6}^{2 / 3}\right]<S>} \\
< & \times \frac{1}{x(1-x)}\left(\frac{<Z>}{\Delta Z}\right)^{2} \operatorname{ergscm}^{-1} \mathrm{~s}^{-1} \mathrm{~K}^{-1}
\end{aligned}
$$




$$
\begin{aligned}
& \times \int_{0}^{1} d\left(\frac{k}{2 k_{F}}\right)\left(\frac{k}{2 k_{F}}\right)^{5} \frac{S\left(k / 2 k_{F}\right)}{\left[\left(k / 2 k_{F}\right)^{2} \epsilon\left(k / 2 k_{F}, 0\right)\right]^{2}} \\
& \equiv<S_{-1}>-\frac{1.018\left(\frac{Z_{1}}{A_{1}} X_{1}+\frac{Z_{2}}{A_{2}} X_{2}\right)^{2 / 3} \rho_{6}^{2 / 3}}{1+1.018\left(\frac{Z_{1}}{A_{1}} X_{1}+\frac{Z_{2}}{A_{2}} X_{2}\right)^{2 / 3} \rho_{6}^{2 / 3}}<S_{+1}>,
\end{aligned}
$$

where $S\left(k / 2 k_{F}\right)$ is the structure factor for the impurity charges, and $\epsilon\left(k / 2 k_{F}, 0\right.$ is the static dielectric function due to degenerate electrons, $k_{F}$ being the electron Fermi wavenumber.

The results (53) and (54) nicely reproduce the experimentally verified rules. The property that the resistivity is proportional to $x(1-x)$ is Nordheim's rule; the property that the resistivity is proportional to $(\Delta Z)^{2}$ is Linde's rule (Ziman 1960).

In order to calculate the structure factor $S(k)$ for the impurity charges, we employ a simple model. We adopt the ion-sphere model, and assume the following radial distribution function for the impurity charges :

$$
g(r)= \begin{cases}0, & \text { for } 0 \leq r \leq a \\ 1, & \text { for } r>a\end{cases}
$$

where $a$ is the ion-sphere radius

$$
a=\left\{3 /\left[4 \pi\left(n_{1}+n_{2}\right)\right]\right\}^{1 / 3} .
$$

Then the structure factor $S(k)$ is obtained as

$$
\begin{aligned}
& S(k)=1+\left(n_{1}+n_{2}\right) \int d^{3} r[g(r)-1] \exp (-i \mathbf{k} \cdot \mathbf{r}) \\
& =1-\frac{3}{(k a)^{3}}(\sin k a-k a \cos k a) .
\end{aligned}
$$

We notice that

$$
S(k) \rightarrow \frac{1}{10}(k a)^{2}, \text { as } k \rightarrow 0 \text {. }
$$

Since the screening effect due to electrons is negligible compared with the effect of the impurity charge correlation expressed by the structure factor (58), we set $\epsilon\left(k / 2 k_{F}, 0\right)=1$ in equation (55). We further replace the structure factor (58) by a simplified form

$$
S^{\prime}(k)=\frac{(k a)^{2}}{(k a)^{2}+10},
$$


Itoh: Transport processes in dense stellar plasmas

which satisfies the condition (59). Insertion of this into equation (55) gives

$$
\begin{aligned}
& <S_{-1}>=\frac{1}{2} \ln \left[1+\frac{2}{5}\left(k_{F} a\right)^{2}\right], \\
& <S_{+1}>=\frac{1}{2}-\frac{5}{4} \frac{1}{\left(k_{F} a\right)^{2}} \ln \left[1+\frac{2}{5}\left(k_{F} a\right)^{2}\right] .
\end{aligned}
$$

The electron Fermi wavenumber $k_{F}$ is written as

$$
k_{F}=2.613 \times 10^{10}\left(\frac{Z_{1}}{A_{1}} X_{1}+\frac{Z_{2}}{A_{2}} X_{2}\right)^{1 / 3} \rho_{6}^{1 / 3} \mathrm{~cm}^{-1} .
$$

The ion-sphere radius $a$ is written as

$$
a=0.7346 \times 10^{-10}\left(\frac{X_{1}}{A_{1}}+\frac{X_{2}}{A_{2}}\right)^{-1 / 3} \rho_{6}^{-1 / 3} \mathrm{~cm} .
$$

Thus we have

$$
k_{F} a=1.920\left(Z_{1} \frac{X_{1}}{A_{1}}+Z_{2} \frac{X_{2}}{A_{2}}\right)^{1 / 3}\left(\frac{X_{1}}{A_{1}}+\frac{X_{2}}{A_{2}}\right)^{-1 / 3} .
$$

It is straightforward to generalize the results in the above to higher component systems. Let the mass fraction of the $\left(Z_{i}, A_{i}\right)$ component be $X_{i}\left(\sum_{i} X_{i}=1\right)$. The result is

$$
\begin{aligned}
& \sigma=8.693 \times 10^{21} \rho_{6}\left(\sum_{i} \frac{X_{i}}{A_{i}}\right) \frac{1}{\left[1+1.018\left(\sum_{i} \frac{Z_{i}}{A_{i}} X_{i}\right)^{2 / 3} \rho_{6}^{2 / 3}\right]<S>} \\
& x \frac{<Z\rangle^{2}}{\left\langle(\Delta Z)^{2}\right\rangle^{-1}}, \\
& \kappa=2.363 \times 10^{17} T_{8} \rho_{6}\left(\sum_{i} \frac{X_{i}}{A_{i}}\right) \\
& \times \frac{1}{\left[1+1.018\left(\sum_{i} \frac{Z_{i}}{A_{i}} X_{i}\right)^{2 / 3} \rho_{6}^{2 / 3}\right]\langle S\rangle} \frac{\langle Z\rangle^{2}}{\left\langle(\Delta Z)^{2}\right\rangle} \\
& \operatorname{ergscm}^{-1} \mathrm{~s}^{-1} \mathrm{~K}^{-1}, \\
& <Z>\equiv \sum_{i} x_{i} Z_{i} \\
& <(\Delta Z)^{2}>\equiv \sum_{i} x_{i}\left(Z_{i}-<Z>\right)^{2} \text {, }
\end{aligned}
$$




$$
x_{i} \equiv \frac{n_{i}}{\sum_{j} n_{j}}
$$

The factor $\langle S\rangle$ is given by equations $(55),(61),(62)$ with

$$
k_{F} a=1.920\left(\sum_{i} Z_{i} \frac{X_{i}}{A_{i}}\right)^{1 / 3}\left(\sum_{i} \frac{X_{i}}{A_{i}}\right)^{-1 / 3} .
$$

\subsection{Concluding remarks}

We have reviewed the recent developments in the field of the transport properties of dense matter. The modern calculations of the transport properties of dense matter make full use of the recent developments in statistical physics, plasma physics, liquid state physics, and solid state physics. Interparticle correlations play essential roles in the quantitative evaluation of the transport properties of dense matter. Now the time is ripe for the close examinations of our understanding of the physics of dense matter by using the observational data of the asteroseismology of white dwarfs.

\subsection{Appendix}

In this Appendix we directly insert equation (58) into eqation (55) and carry out the integration. The result is

$$
\begin{aligned}
& <S_{-1}>=3 \sum_{n=0}^{\infty}(-1)^{n} \frac{n+2}{n+1} \frac{\left(2 k_{\mathrm{F}} a\right)^{2 n+2}}{(2 n+5) !} \\
& <S_{+1}>=\frac{1}{2}-\frac{3}{4} \frac{1}{\left(k_{\mathrm{F}} a\right)^{2}}\left[1-\frac{\sin \left(2 k_{\mathrm{F}} a\right)}{2 k_{\mathrm{F}} a}\right] .
\end{aligned}
$$

The differences between the $\langle S\rangle$ values calculated from eqations (61) and (62) and those calculated from equations (72) and (73) are typically 10 $\%$ - $20 \%$ (the latter is generally larger).

The structure factor (58) based on the ion-sphere model is an approximation of the real ionic correction, and its oscillatory behavior does not have a rigorous meaning. Thus, in this paper, we will adopt the smooth structure factor (60) which leads to a simpler expression for $\left\langle S_{-1}\right\rangle$. In any case, we should bear in mind that the differences in the $\langle S\rangle$ values caused by the use of the different structure factors indicate the accuracy of the present calculation. 


\subsection{References}

Bradley, P.A., \& Winget D.E. 1991, ApJS, 75, 463

Bradley, P.A., Winget, D.E., \& Wood, M.A. 1992, ApJ, 391, L33

Coldwell-Horsfall, R.A., \& Maradudin, A.A. 1960, J. Math. Phys., 1, 395

Flowers, E., \& Itoh, N. 1976, ApJ, 206, 218

Flowers, E., \& Itoh, N. 1981, ApJ, 250, 750

Itoh, N., Hayashi, H., \& Kohyama, Y. 1993, ApJ, in press

Itoh, N., \& Kohyama, Y. 1993, ApJ, 404, 268

Itoh, N., Kohyama, Y., Matsumoto, N., \& Seki, M. 1984a, ApJ, 285, 758;

erratum 404, 418

Itoh, N., Kohyama, Y., \& Takeuchi, H. 1987, ApJ, 317, 733

Itoh, N., Matsumoto, N., Seki, M., \& Kohyama, Y. 1984b, ApJ, 279 , 413

Itoh, N., Mitake, S., Iyetomi, H., \& Ichimaru, S. 1983, ApJ, 273, 774

Iyetomi, H., \& Ichimaru, S. 1982, Phys. Rev. A, 25. 2434

Jancovici, B. 1962, Nuovo Cimento, 25, 428

Ogata, S., \& Ichimaru, S. 1987, Phys. Rev. A, 36, 5451

Pollock, E.L., \& Hansen, J.P. 1973, Phys. Rev. A, 8, 3110

Raikh, M.E., \& Yakovlev, D.G. 1982, Ap. Space Sci., 87, 193

Yakovlev, D.G., \& Urpin, V.A. 1981, Soviet Astr., 24, 303

Ziman, J. 1960, Electrons and Phonons (Oxford Univ. Press) 